\title{
Long-Term Inhibition of Kindled Seizures by Brain Stimulation
}

\author{
Jiao Shao and Elliot S. Valenstein ${ }^{\prime}$ \\ Departments of Psychology, Beijing University. Beijing. The People's Republic of China. \\ and The University of Michigan, Ann Arbor, Michigan 48109
}

\author{
Received November 17, 1981
}

\begin{abstract}
It was concluded from previous studies that prior stimulation produced a longlasting inhibition of kindled seizures only when a series of convulsions were evoked. The present study describes a brain stimulation procedure capable of producing a long-lasting inhibition of kindled convulsions without evoking prior convulsions of EEG afterdischarge. Electrodes were implanted in the amygdala of rats, which were then kindled to a criterion of five successive tonic-clonic convulsions. After kindling, the animals were exposed to a regimen of intermittent amygdala stimulation, which was gradually increased in intensity in small incremental steps from a point just below the convulsion threshold to an intensity above the original kindling current. Using this procedure, it proved possible to administer high stimulation intensities without evoking any behavioral convulsions or EEG afterdischarges. Subsequent to this inhibitory procedure, amygdala stimulation did not evoke convulsions or EEG seizures for as long as 7 days. Additional studies tested the relative effectiveness of different stimulation regimens and parameters for producing long-lasting inhibition of kindled seizures. The magnitude of the incremental intensity steps, the interstimulus interval, and the intensity of stimulation all proved to be relevent. We concluded that long-lasting inhibition of kindled seizures could be produced by prior stimulation that did not evoke seizures.
\end{abstract}

\footnotetext{
Abbreviations: CT-convulsion threshold, IBS-inhibitory brain stimulation, ER-emotional reponse, EEG--electroencephalogram.

${ }^{1}$ This work was supported by National Science Foundation grant BNS 8025468. The authors gratefully acknowledge the technical assistance of Buda Martonyi, the helpful comments from Terry Robinson and Alfred Mansour, construction of figures by Judith Lehman, and typing of the manuscript by Judy Baughn. Israel Lieblich demonstrated the emotional response test. Professor Shao is a Visiting Scholar participating in the Exchange Program between the Departments of Psychology of Beijing University and the University of Michigan. Please address correspondence to Professor Elliot $S$. Valenstein, Neuroscience Laboratory Building, 1103 E. Huron, University of Michigan, Ann Arbor, Mich. 48109.
} 


\section{INTRODUCTION}

The gradual development of afterdischarges and behavioral convulsions in response to a regimen of brief, electrical stimulations of the amygdala and other brain structures has been referred to as the kindling phenomenon (4). During kindling, a brain stimulus which initially evoked only a brief electrical afterdischarge, gradually starts to evoke progressively longer afterdischarges and more extensive and long-lasting tonic-clonic convulsions. After an animal has undergone the kindling process, a brain stimulus will normally evoke a convulsion even after long intervals without stimulation. Kindling has been used extensively as a model of epilepsy and to a somewhat lesser extent, to study neuronal plasticity [see (9) for a review]. Although the processes underlying the gradual facilitation of the response to a brain stimulus were studied primarily, the fact that there was also an "inhibitory" aspect to kindling was recognized quite early. Goddard et al. (5), for example, reported that kindled convulsions do not develop if stimulation was administered in massed trials with interstimulus intervals less than 10 min. Those authors also observed in animals already "kindled" that " continuous stimulation" which evoked several convulsions blocked the response to a normally effective stimulus for periods lasting to 3 to 5 days.

More recently, several investigators studied the inhibitory effect of brain stimulation in kindled animals in more detail. Both Mucha and Pinel (10) and Sainsbury et al. (13) reported a short- and long-term inhibition after stimulation to the amygdala and hippocampus, respectively. Short-term inhibition was seen only after prior stimulation that produced an afterdischarge. The inhibition was more pronounced after a motor convulsion than after stimulation which evoked only an afterdischarge. At its maximum, the short-term inhibition consisted of a total suppression of afterdischarge and convulsions to stimulation administered less than $10 \mathrm{~min}$ after a preceding stimulation. After 70 to 90 min no evidence of inhibition was observed.

The long-term inhibition was evident only after a series of motor convulsions had been evoked. As described by Mucha and Pinel (10), longterm inhibition occurred after 19 amygdala stimulations (delivered at 1.5$\mathrm{h}$ intervals) almost all of which evoked stage 5 convulsions. When stimulated as much as 4 days later, both the afterdischarge and the severity of the convulsions were significantly attenuated. Sainsbury et al. (13) found an inhibition evident $24 \mathrm{~h}$ after five successive stimulations and convulsions, but in their experiment there was no decrease in the severity of the convulsions, only a shortened duration. Comparing their results with Mucha and Pinel's, Sainsbury et al. concluded that "the longer lasting inhibition may be due to the cumulative effects of the prior motor seizures" [(13), 
p. 487]. Using the electroconvulsive shock paradigm, Essig and Flanary (3) also reported that a number of prior convulsions cumulatively increased resistance to the induction of a subsequent convulsion.

Thus, earlier studies reported that stimulation must at least produce an afterdischarge in order to result in any detectable inhibition even for short periods less than $90 \mathrm{~min}$ in duration. It was concluded that a long-term inhibition lasting several days resulted from the cumulative effect of a series of motor convulsions. In contrast, we report here a procedure that produces a long-term inhibition after a regimen of stimulation that does not evoke either motor convulsions or, as far as could be determined, any afterdischarge.

\section{METHODS}

Subjects. Fifteen mature ( 300 to $\mathbf{4 0 0} \mathrm{g}$ ), male hooded rats of the LongEvans strain (Simonsen Co., Gilroy, CA) served as the subjects for the major portion of the results described. An additional six male rats of the same strain, weighing 250 to $300 \mathrm{~g}$, were used in supplementary experiments.

Electrode Implantation. Bipolar electrodes were stereotaxically implanted in the left and right amygdala under chloral hydrate (Choloropent, Fort Dodge Co., Fort Dodge, IA) anesthesia. With the skull level the coordinates were $2.5 \mathrm{~mm}$ posterior to bregma, $4.5 \mathrm{~mm}$ lateral, and $8.5 \mathrm{~mm}$ below the dorsal surface of the skull. The electrodes consisted of two twisted, Teflon-coated, stainless-steel wires ( $200 \mu \mathrm{m}$ diameter, $280 \mu \mathrm{m}$ with coating) soldered to gold-plated microminiature connectors (Winchester Co. 8456). An additional connector attached to a skull screw was placed anterior to the coronal suture and served as a ground for the electroencephalogram (EEG) recording. Cranioplastic cement was used to attach the electrode assembly to the skull.

Stimulation and Recording. Stimulation was delivered by a constant current source and in all procedures consisted of a $60-\mathrm{Hz}$ sine wave, $1-\mathrm{s}$ duration train. Only one of the amygdala electrodes was used for stimulation in any animal. A polygraph (Grass Model 7) and two wide band AC EEG preamplifiers (Grass Model 7P5) were used to record from both amygdala simultaneously.

Kindling Procedure. After recovery from surgery, the animals were stimulated once daily with a $50-\mu \mathrm{A}$ current. The EEGs from both electrodes were obtained prior to stimulation (baseline) and for at least $60 \mathrm{~s}$ after the stimulation. Convulsions were rated on a scale modified from that used by Racine (12) as follows: $(0)$ no afterdischarge, $(0+)$ afterdischarge with no behavioral concommitants, (1) mouth and facial movements, (2) head nodding, (3) forelimb clonus, (4) tonic-clonic convulsion with rearing, (5) 
tonic-clonic convulsions with rearing and falling. The animals were stimulated daily until they exhibited five successive stage 5 convulsions.

Convulsion Threshold Determinations. Two to four weeks after the 15 rats had attained the criterion of five successive stage 5 convulsions, a convulsion threshold (CT) was determined using the same stimulus parameters described above except current intensity was varied. The $\mathrm{CT}$ was determined by gradually reducing the intensity of the stimulating current below the $50 \mu \mathrm{A}$ used during kindling. First, all animals were stimulated at $25 \mu \mathrm{A}$ and on the next day at $16 \mu \mathrm{A}$. Stimulation at both intensities evoked stage 5 convulsions in all animals. The current intensity was then reduced by $2 \mu \mathrm{A}$ each day until no convulsions were evoked. The current was then raised $2 \mu \mathrm{A}$ and in every instance a stage 5 convulsion was triggered. This latter current was considered to be the CT. The reliability of the CT to evoke a stage 5 convulsion was determined on three consecutive days.

Inhibitory Brain Stimulation Procedure. The inhibitory brain stimulation (IBS) procedure consisted of the following regimen. The animals received 20 consecutive stimulations ( 1 -s duration; 6.5 -s interstimulus interval) at an intensity 2 to $4 \mu \mathrm{A}$ less than the CT. After the 20th stimulation, two stimulations at an intensity $2 \mu \mathrm{A}$ higher were administered using the same stimulus duration and interstimulus interval. The stimulus intensity was increased in $2 \mu \mathrm{A}$ steps, with two repetitions at each step until an animal either had a convulsion (always a stage 5 convulsion) or an intensity of $54 \mu \mathrm{A}$ was used. It should be noted that $54 \mu \mathrm{A}$ is higher than the intensity used for kindling. If an animal had a convulsion, the procedure was repeated each day until $54 \mu \mathrm{A}$ was used without eliciting a convulsion. Therefore, a completed IBS series refers to a gradually increasing regimen of stimulus intensities from below the convulsion threshold to $54 \mu \mathrm{A}$ without the occurrence of a convulsion. In a few animals this could be accomplished on the first attempt, but in most cases it required several daily stimulation trials. The EEGs were obtained prior to stimulation and immediately after each brain stimulus was administered.

Recovery of Seizure Susceptibility After Inhibitory Brain Stimulation. To determine the time course of the recovery of seizure susceptibility, the animals were stimulated at their CT intensity at different intervals after completion of the IBS procedure. Initially, the animals were stimulated at different intervals to $24 \mathrm{~h}$ after completion of the IBS procedure, but as no animal exhibited any EEG afterdischarge or behavioral response to stimulation at the CT, the interval was gradually extended beyond $24 \mathrm{~h}$ and to as long as 8 days in the case of one animal. Other details of these "recovery tests" are described together with the results. After a seizuresusceptibility recovery curve was obtained, the animals had a second IBS series using the procedure described above. 
Emotional Response Test. As several animals were very difficult to handle after an inhibitory brain stimulation series, we tested the reactivity of all animals using an emotional response (ER) scale. The ER scale was identical to that used recently to test animals after septal ablations (8) and it in turn was modified from an emotionality scale used earlier (1). The sca'e consists of the following measures: (i) resistance to capture (using a dorsal approach); (ii) resistance to handling; (iii) vocalization; (iv) biting; (v) reflexive head jerk and vibrissae stiffening upon presentation of an object to the rat's mouth; (vi) startle response to the experimenter blowing on top of rat's head; (vii) urination and defectation; and (viii) muscle tension. Items (i) through (vi) were each scored 0-3; items (vii) and (viii) were scored 0 or 1 ; the maximal possible ER score was 20 . Rats with septal ablations commmonly receive ER scores between 10 and 17 immediately after the lesion and they gradually become less emotional, commonly achieving scores around 5 two weeks after the ablation (8). The average laboratory rat that is accustomed to handling usually gets a score between 0 and 3.

Histology. At the completion of the experiment, the animals were killed with an overdose of sodium pentobarbital and after perfusion the brains were stored in Formalin. Wet frozen sections $(80 \mu \mathrm{m})$ were projected and the electrodes were localized and recorded on stereotaxic maps.

\section{RESULTS}

Kindling and Convulsion Threshold. The average number of stimulations required to reach the first stage 5 convulsion was 7.3 for the 15 animals. With the exception of one animal, all animals had stage 5 convulsions in response to every stimulation after they had the first stage 5 convulsion. After the animals had five successive stage 5 convulsions, the $\mathrm{CT}$ was determined. The range of $\mathrm{CT}$ for the 15 rats was 4 to $14 \mu \mathrm{A}$ with a median score of $8 \mu \mathrm{A}$. No relationship was evident between the CT and the rate of kindling. Typically, an "all-or-none" response was observed at a given current as the animals either had a stage 5 convulsion or no EEG afterdischarge or behavioral response was evident. Moreover, the duration of the stage 5 convulsion evoked by a $C T$ stimulus intensity did not differ significantly from that evoked by a $50-\mu \mathrm{A}$ stimulus. A finding that should be emphasized is that the CT reliably evoked a stage 5 convulsion on each of the three or more daily stimulations administered to every animal. Thus, the occurrence of a stage 5 convulsion did not influence the response to CT stimulation administered $24 \mathrm{~h}$ later.

Inhibitory Brain Stimulation. Using the IBS procedure described above, it proved possible to stimulate all 15 animals with a 54- $\mu$ A "supra-kindling" intensity without evoking any convulsive behavioral response or EEG af- 


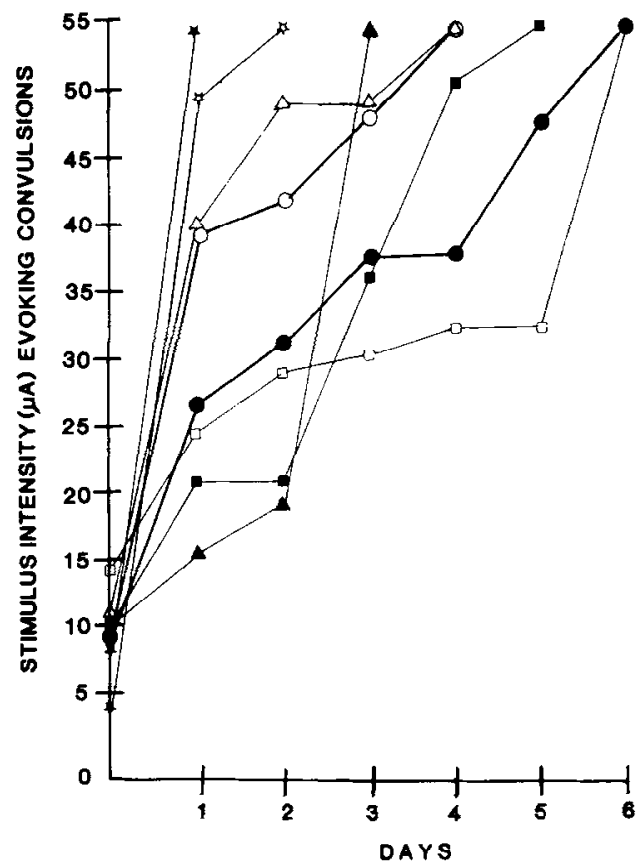

FIG. 1. Progress of six representative animals during daily inhibitory brain stimulation (IBS) trials. Depicted is the stimulus intensity evoking a motor convulsion except at $54 \mu \mathrm{A}$, the point at which the test was terminated. The average progress of all 15 animals during their first and second IBS series is also shown (see text for details). The point at 0 days represents the convuision threshold. Rats: $875(\star), 838(\star)), 871(\triangle), 869(\triangle), 876(\sigma)$, and $873(\square)$. Average $(N=15)$ of first IBS $(\bullet)$, second IBS (O).

terdischarge. In the case of two animals, the current intensity could be raised to $54-\mu \mathrm{A}$ without evoking a convulsion on the first day the IBS procedure was administered. Usually, however, it was necessary to repeat the IBS procedure for several days as the animals had a stage 5 convulsion in response to stimulation at an intensity below $54 \mu \mathrm{A}$. In all cases, the animals that had a convulsion at a given intensity of stimulation always tolerated a higher intensity of stimulation the next day. The median number of days required to complete the IBS procedure was 4 , but two animals needed 6 days. No relationship between the number of days required to complete the IBS procedure and the CT was evident. Figure 1 illustrates the progression of the IBS procedure with days in six animals and also presents the average progression curve for all 15 animals. The results are expressed in terms of the intensity of stimulation that could be administered before a convulsion was evoked. Thus, in Fig. 1, rat 871 had a CT of 10 $\mu \mathrm{A}$ and on the first day the IBS procedure was used an intensity of 15 
$\mu \mathrm{A}$ evoked a stage 5 convulsion. On the second day, an intensity of 18 $\mu \mathrm{A}$ evoked a convulsion, but on day 3 it proved possible to administer a 54- $\mu \mathrm{A}$ stimulus without evoking a convulsion. In exploratory work, we were able to raise the current to $100 \mu \mathrm{A}$ without producing a convulsion, but we have not as yet systematically explored the upper limits of the IBS procedure.

After the animals had been tested for recovery of their sensitivity to stimulation (results described below), the IBS procedure was repeated. There was a tendency for the animals to exhibit the same pattern they had shown initially so that those which attained $54 \mu \mathrm{A}$ either faster or slower than average tended to continue to be faster or slower, respectively. In general, however, the animals required fewer trials to attain $54 \mu \mathrm{A}$ than they did initially and this is reflected in the average curve of the second IBS depicted in Fig. 1. In the second IBS session, seven animals could be stimulated at $54 \mu \mathrm{A}$ the first day without exhibiting any convulsion compared with only two animals on the first IBS session. The median number of days required to attain an intensity of $54 \mu \mathrm{A}$ without convulsions was less than two for the second IBS session compared with four for the first IBS session. Prior to beginning the second IBS series, the CT of the 15 rats was reassessed. In 10 animals the $\mathrm{CT}$ was unchanged, but for five of the animals the CT was found to be from 2 to $4 \mu \mathrm{A}$ lower.

In most animals no EEG afterdischarge was evident during the IBS procedure either during the 6.5-s interstimulus intervals or after the 54$\mu \mathrm{A}$ intensity was reached. It was observed, however, that the EEG pattern was often modified from the prestimulation baseline record. The modification could take several forms, but most typically it was characterized by a reduction in the amplitude and frequency of the EEG pattern that lasted approximately $2 \mathrm{~min}$ after stimulation at $54 \mu \mathrm{A}$, before returning to the baseline pattern. In some cases after the $54-\mu \mathrm{A}$ stimulation, the EEG record became almost completely flat for about $20 \mathrm{~s}$ followed by a 10 to $20 \mathrm{~s}$ period when what appeared to be interictal spiking might occur. It should be noted that no changes in the EEG record from the contralateral amygdala electrode was observed at these times. In two cases, afterdischarge lasting to 12.5 and $15 \mathrm{~s}$, respectively, was evoked by stimulation at intermediate intensities, but the afterdischarge ended abruptly and was not accompanied by any convulsive behavior (see Fig. 2). With the exception of these two cases, afterdischarge was not observed during the IBS series either because stimulation at 6.5 -s intervals prevented its occurrence or because a very brief afterdischarge could not be recorded during the 3 to $4 \mathrm{~s}$ immediately after stimulation when the amplifiers were sometimes blocked. Figure 2 illustrates typical EEG records during different stages of the IBS procedure. 

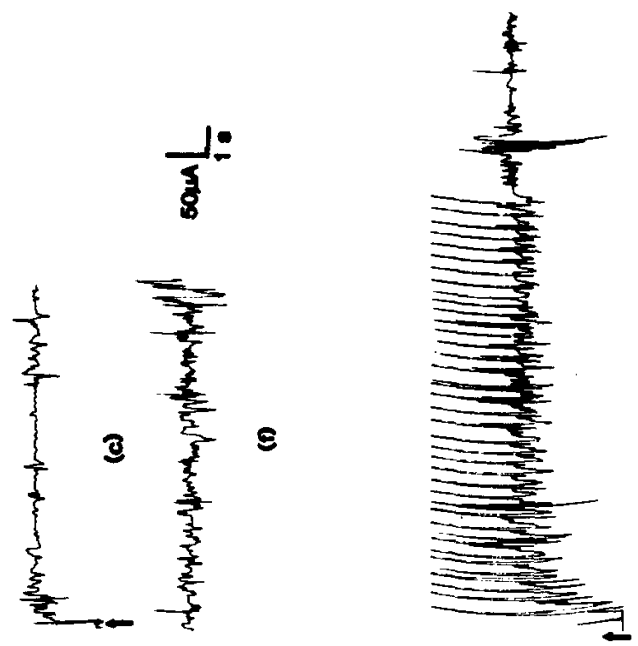

3
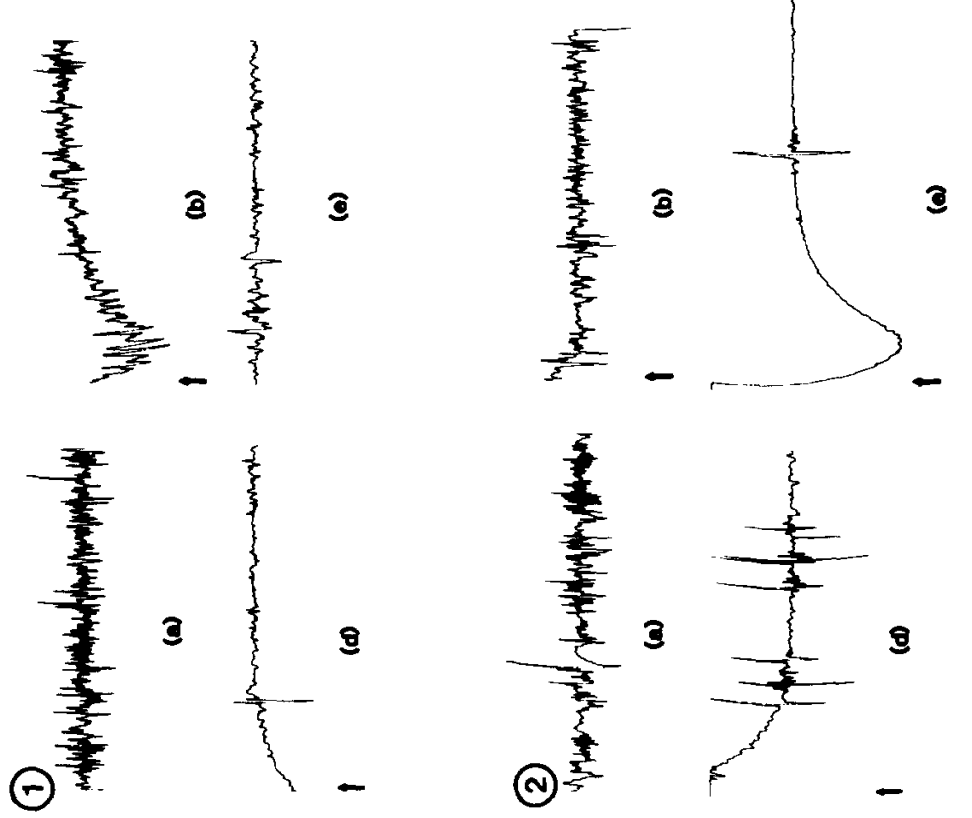

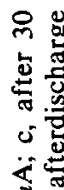

$\infty$ 동

过

ส

0

iิ

동

路

จิ

1 늠

$\infty<=$

垫

政

。迹焉

छ匹

E

다

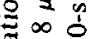

苛吉

荡

$\Xi \dot{0}$

政

要

( ) : 至

$\infty \vdots \nabla$

혼

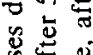

究

8 is

8

政要

空苍

要薄

인

8 氙造

$\$ \infty$

要

낭

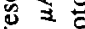

岩范

吕

N

站方 
Although our IBS regimen of gradually increasing intensities usually did not evoke any motor convulsions or afterdischarges, there was frequently behavioral evidence that the stimulation was effective. Most commonly, the animals showed contralateral turning or contralateral forepaw lifting during the stimulation and some started to engage in a "face washing" response with their forepaws during the stimulation and for a brief period afterward.

After the IBS procedure, a few animals exhibited a very marked increase in reactivity similar to that frequently seen immediately after a stage 5 convulsion. These animals resisted handling, were often very aggressive and they commonly vocalized when they were being picked up. We tested the 15 animals with the emotionality response (ER) test described above in the Methods section.

Although the results were variable and the animals did not always seem more emotional after the IBS was completed, the post-IBS emotionality of several of them was comparable to that seen after septal ablations. These results are summarized in Table 1 . When the tests were more than 3 min after completion of the IBS procedure, the ER scores were no longer elevated. It was our impression that the heightened emotionality occurred more frequently when the animals were tested during a period of interictal spiking which sometimes followed the flattened EEG record seen after completion of an IBS series.

TABLE 1

Distribution of Emotional Response Scores

\begin{tabular}{cccccccc}
\hline & \multicolumn{8}{c}{ Scores } \\
\cline { 2 - 8 } & $0-2$ & $3-5$ & $6-8$ & $9-11$ & $12-14$ & $15-17$ & $18-20$ \\
\hline Number of rats & 3 & 3 & 3 & 1 & 1 & 0 & 4 \\
\hline
\end{tabular}

Recovery of Seizure Susceptibility. After completion of an IBS series, all 15 animals exhibited a long-lasting reduction in responsiveness to stimulation at their CT intensity. The duration of the recovery period varied between animals, but none exhibited a stage 5 convulsion when stimulated at the CT intensity prior to $48 \mathrm{~h}$ after completion of the IBS series. Only one animal had any afterdischarge after stimulation prior to $48 \mathrm{~h}$ after completion of the IBS series; this animal (872) had a stage 2 convulsion after a 36-h interval. The median time interval between the completion of the IBS series and the evoking of a stage 5 convulsion by CT stimulation was $72 \mathrm{~h}$. One animal required 8 recovery days after the IBS procedure before a stage 5 convulsion could be evoked. The individual differences in 


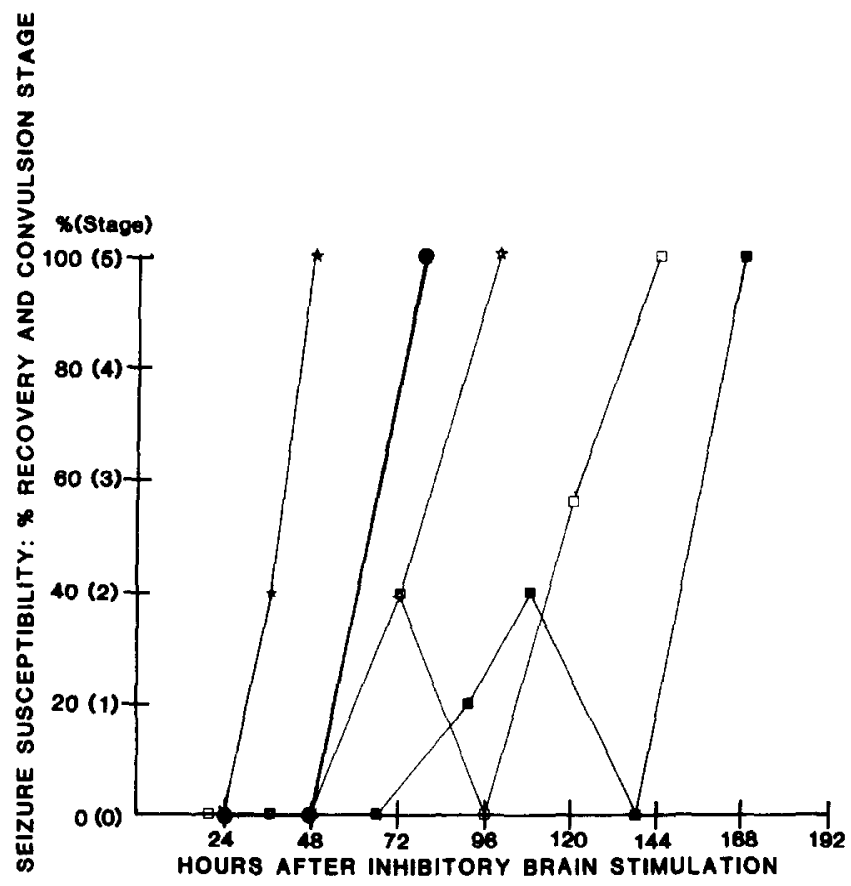

FIG. 3. Recovery of seizure susceptibility after a single inhibitory brain stimulation series in the four animals that exhibited a gradual recovery. None of the other 11 animals exhibited any intermediate responses before they had a stage 5 convulsion. The average response of the 11 animals that recovered abruptly is also illustrated. Recovery of seizure susceptibility was determined by testing animals at their convulsion threshold intensity. Rats: $872(\star), 867$ $(\$), 876(\square)$, and $877(\square)$; average $(N=11)$, $\bullet$. Note: In quantifying percent recovery of seizure susceptibility, we arbitrarily assumed that the convulsive stages are equidistant from each other.

time required to recover seizure susceptibility was not related either to the CT intensity or the number of IBS days required to complete the series.

In the majority $(N=11)$ of cases, the animals exhibited no behavior or EEG response prior to the time that a stage 5 convulsion was evoked. When a stage 5 convulsion was evoked after an IBS series, its duration did not differ from that observed during determination of the CT. In four rats, however, some EEG afterdischarge and either a stage 1 or 2 convulsion was evoked between 36 and $136 \mathrm{~h}$ after the IBS series, prior to the time a stage 5 convulsion could be evoked. On several occasions, rats that showed intermediate convulsive behavior on one day exhibited no EEG or behavioral response to $\mathrm{CT}$ stimulation on the next day. The results from the four rats that exhibited intermediate responses are shown on Fig. 3 with the average curve for the 11 subjects that showed an abrupt recovery of seizure susceptibility with no intermediate response prior to the time a stage 5 
convulsion was evoked. When recovery of seizure susceptibility was determined after the second IBS series, no animal showed any intermediate response. The majority of animals $(N=9)$ exhibited a stage 5 convulsion $72 \mathrm{~h}$ after completion of the IBS series, while the remainder $(N=6)$ had a stage 5 convulsion after $48 \mathrm{~h}$.

The IBS series always involved a gradual raising of the stimulus intensity from just below the CT to an intensity of $54 \mu \mathrm{A}$. It was not clear, therefore, whether or not the long-term inhibition we observed could have been obtained by stimulating animals only at intensities below the CT intensity. To answer this question, three animals received 80 stimulations at 6.5 -s interstimulus intervals at an intensity $2 \mu \mathrm{A}$ below their CT. This number of stimulations was greater than that received by any animal during the IBS procedure. When tested $60 \mathrm{~min}$ later at the CT intensity, all three animals had a stage 5 convulsion. Thus, we concluded that stimulation at suprathreshold intensities was necessary to achieve long-term inhibition although we have not determined whether or not it is necessary to raise the intensities of the IBS as high as $54 \mu \mathrm{A}$.

As our determination of the recovery of seizure susceptibility was made by stimulating animals at their CT intensities, some of the rats were tested later with a suprathreshold intensity. We tested six rats with a $50-\mu \mathrm{A}$ stimulus administered every $30 \mathrm{~min}$ after completion of an IBS series, and six other rats were tested on the same schedule after a stage 5 convulsion evoked by stimulation at an intensity just above the CT. The six animals that received an IBS series had a stage 5 convulsion at a mean of $80 \mathrm{~min}$ (range 50 to 130) later, whereas the six animals that were tested after a stage 5 convulsion had their second stage 5 convulsion at a mean of 50 min (range 30 to 120) afterward. All animals had either a stage 5 convulsion or they displayed no convulsive behavior or electrographic evidence of afterdischarge. Thus, when tested with a suprathreshold intensity only a short-term inhibition was observed after IBS and the duration of the inhibition was only slightly greater than that seen after a stage 5 convulsion.

Supplementary Experiments. Although only a relatively short-lasting inhibition could be demonstrated with a suprathreshold intensity after a single IBS series, it was thought that a regimen of IBS series repeated daily might have a cumulative effect. To test this possibility, six additional rats were tested in a second experiment. They were also used later to test the importance of the interstimulus interval used during our IBS series. These animals were implanted with electrodes in the amygdala with the procedure described above and were kindled to a criterion of five successive stage 5 convulsions. After the animal attained the kindling criterion and then completed a single IBS series, the IBS procedure was repeated during 5 to 9 consecutive days in different animals. After the extended IBS series, 


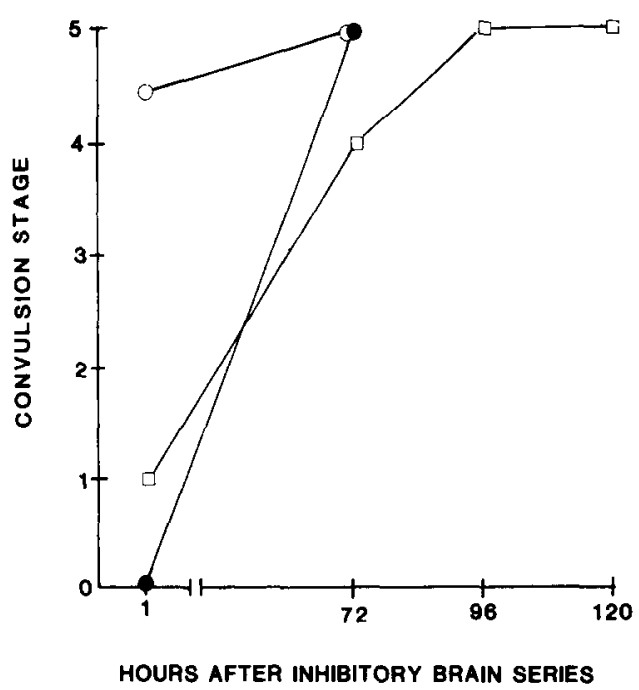

FIG. 4. Recovery of seizure susceptibility after a daily regimen of an inhibitory brain stimulation (IBS) series. Animals were tested with a suprathreshold current intensity (50 $\mu \mathrm{A})$ at $1,72,96$, and $120 \mathrm{~h}$ after completion of the IBS series. 15G: 1 IBS (O), 9 IBSs (प); 20G: 7 IBSs (๑).

the animals were tested with a suprathreshold current of $50-\mu \mathrm{A}$ intensity at $1,72,96$, and $120 \mathrm{~h}$ after completion of the last IBS series. Three were then retested later after a single IBS series.

The results were very systematic and provided evidence that the effect of a daily regimen of IBS was cumulative. After a series of five or more IBS, there was only a brief (less than $25 \mathrm{~s}$ ) afterdischarge and either no evidence of a motor convulsion or only a stage 1 convulsion when tested $1 \mathrm{~h}$ later with a $50-\mu \mathrm{A}$ stimulus. Evidence of a residual inhibitory effect manifested by an attenuated EEG and behavioral response could be detected $72 \mathrm{~h}$ later in most animals and up to $96 \mathrm{~h}$ after the extended IBS series in three animals. During the post-IBS period, the afterdischarge produced by $50-\mu \mathrm{A}$ stimulation gradually lengthened as a function of time in parallel with the gradually increasing convulsion stage. After a single IBS series, in contrast, the results were more variable and only a relatively small reduction in length of the afterdischarge and convulsion stage was detected at the 1-h interval. The results of two animals are shown in Fig. 4.

As the 6.5-s interstimulus interval during IBS was selected somewhat arbitrarily, we subsequently tested the six animals with different interstimulus intervals while all other aspects of the IBS procedure were unchanged. When a 1.0-s interstimulus interval was used, all animals consistently had a stage 5 convulsion before reaching the $54-\mu \mathrm{A}$ intensity. The median 
intensity that evoked a stage 5 convulsion was $12 \mu \mathrm{A}$. We repeated the test with a 1.0-s interstimulus interval three times with the same results. On alternate days when we used our standard 6.5-s interstimulus interval, all six animals attained $54 \mu \mathrm{A}$ without convulsions. Using a 3.0 -s interstimulus interval, the intensity could be raised to $54 \mu \mathrm{A}$ in $50 \%$ of the six animals without evoking any convulsion. The remaining three animals had stage 5 convulsions at a median intensity of $20 \mu \mathrm{A}$. Using the three animals that had convulsions, the interstimulus interval was extended to $6.0 \mathrm{~s}$ and two animals attained $54 \mu \mathrm{A}$ without convulsions. The remaining animal had a stage 5 convulsion at $32 \mu \mathrm{A}$. The results strongly indicated that the effectiveness of an IBS series was attenuated when interstimulus intervals less than $6.0 \mathrm{~s}$ were used. These results are illustrated in Fig. 5.

Some differences were observed in the responses of the six younger rats tested in the second experiment from those of the older subjects used in

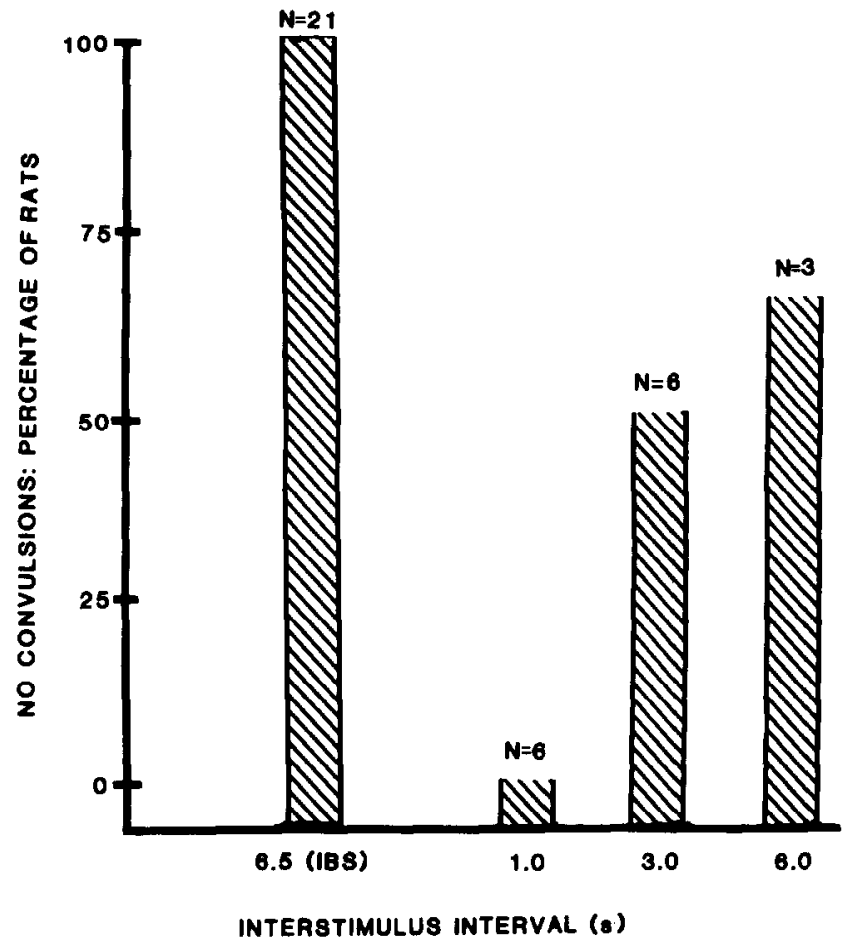

FIG. 5. Percentage of animals in which inhibitory brain stimulation (IBS) could be raised to $54 \mu \mathrm{A}$ without evoking a convulsion as a function of interstimulus interval (see text for details). 


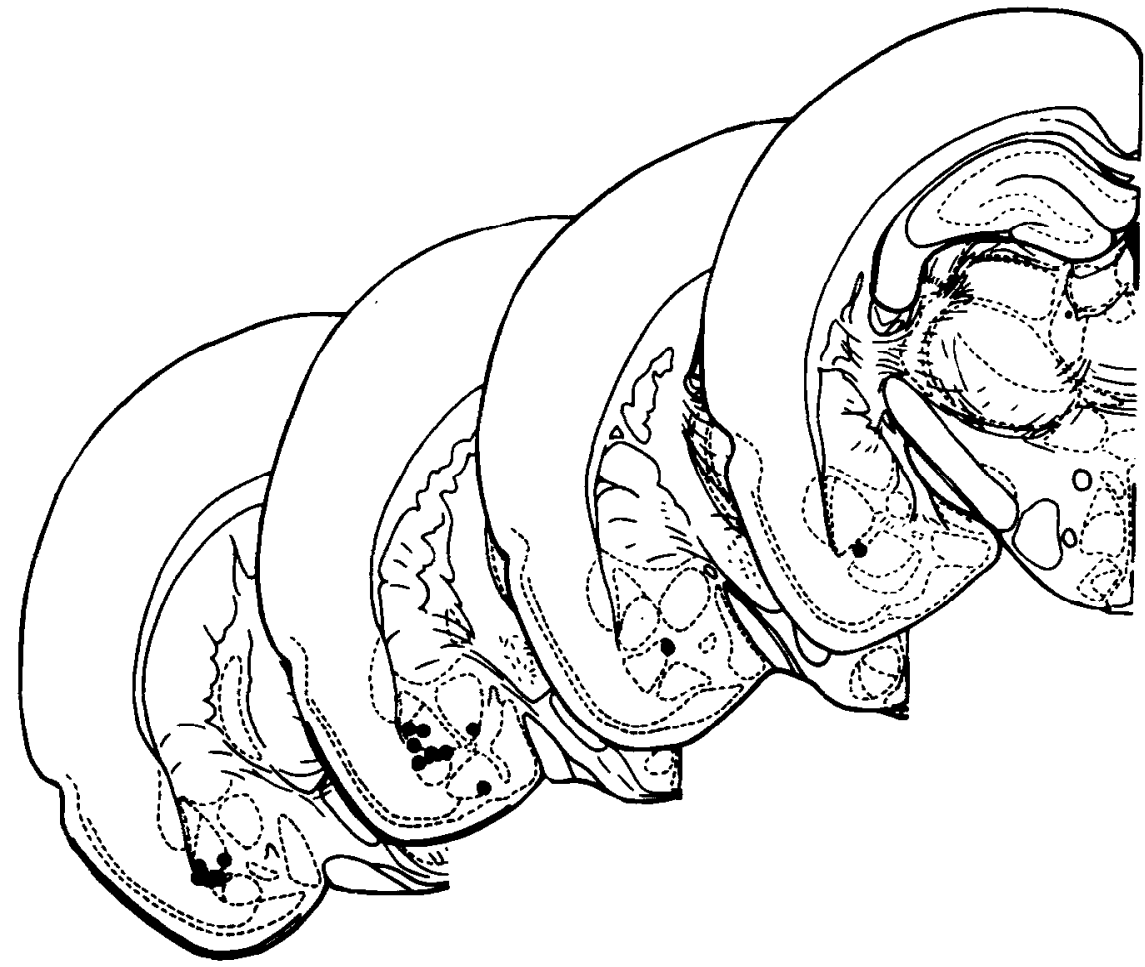

FIG. 6. Electrode tip placements of 15 subjects used in the first experiment. [Adapted from König and Klippel (7).]

the first experiment. For example, during the IBS several of the younger animals exhibited brief ( 2 to $4 \mathrm{~s}$ ) convulsions consisting of clonus of the paws and mouth sometimes while rising on their hind paws. In these instances, the responses resembled the first 2 to $4 \mathrm{~s}$ of a response that normally would progress to a generalized stage 5 convulsion in kindled animals. These responses, however, were rapidly terminated. Such responses usually occurred in the absence of any detectable electrographic afterdischarge, but on a few occasions a short ( 4 to $6 \mathrm{~s}$ ) afterdischarge was evident. The brief convulsive responses tended to occur at some intermediate intensity between the CT and $54 \mu \mathrm{A}$. In a few instances, a stage 4 or 5 convulsion was evoked within a particular intensity range so it was necessary to reduce the incremental steps from 2.0 to $1.0 \mu \mathrm{A}$ throughout that range. It should be emphasized, however, that in the majority of cases no motor convulsive behavior or afterdischarge was observed during the IBS.

Histology. Figure 6 illustrates the sites of the tip of the electrode in the 
initial 15 rats used in the major portion of the experimental studies. All electrodes were located within the amygdala; the majority $(87 \%)$ were placed in the anterior one-third of the extent of this structure. The electrode placements in the second group of six animals were found to be very similar to those shown in Fig. 6.

\section{DISCUSSION}

The present experiment demonstrates that it is possible to obtain a longterm inhibition of kindled seizures by brain stimulation that does not evoke convulsive behavior. When animals are tested at convulsion threshold intensities, our procedure of exposing them to a gradually increasing intensity of brain stimulation resulted in an inhibition of kindled seizures that persisted from 2 to 8 days following a single session of stimulation. After an extended series of daily sessions of increasing stimulation intensities, longlasting inhibition could be demonstrated even when the animals were tested with suprathreshold currents. These data do not support the conclusion drawn from earlier studies $(10,13)$ that long-lasting inhibition of kindled seizures requires a series of motor convulsions.

Using a very different technique, Herberg et al. (6) demonstrated that antecedent stimulation at current intensities below the convulsion threshold could decrease the probability of seizures occurring during subsequent lateral hypothalamic self-stimulation. Those authors concluded that antecedent stimulation could produce a "refractory state" that does not depend on inhibitory feedback from the motor system during the occurrence of a convulsion. The inhibition demonstrated by Herberg et al., however, was detected only at intervals shorter than $2 \mathrm{~h}$ after antecedent subconvulsive stimulation. Moreover, because Herberg et al. did not report any EEG data, it is not clear whether or not the prior "subconvulsive stimulation" in their experiment had evoked any afterdischarge. In contrast, using our stimulation procedure we demonstrated that long-lasting inhibition of seizure propensity can be produced without the occurrence of convulsions, and as far as we can determine, without evoking any afterdischarge.

It has been suggested that the long-lasting inhibition produced by an antecedent series of convulsions may result from depletion of some substance. Apparently, it is necessary that the antecedent stimulation be above the intensity normally sufficient to evoke convulsions as we have shown that a relatively long series of stimulation at intensities below the convulsion threshold did not produce any evidence of long-term inhibition. Using our procedure, however, it is possible to gradually increase the intensity of the antecedent stimulation to many times above the convulsion threshold without evoking convulsions. Although several interpretations are possible, it 
appears that our procedure may be more closely related to an habituation process rather than involving depletion of some biochemical reserves produced by a series of convulsions.

The importance of gradually increasing the intensity of stimulation needs to be underscored as our preliminary attempts to abbreviate the inhibitory process by introducing larger incremental steps always produced a convulsion. What seems to be important in producing the long-term inhibition is the ability to deliver normally convulsant intensities of stimulation under conditions which do not evoke epileptic activity. We accomplished this by a regimen of small incremental increases in current intensities delivered at 6.5-s interstimulus intervals. We do not know yet if this interval is optimal, but it was established in the second experiment that it is difficult to raise the current during successive stimulations without evoking convulsions if the interstimulus interval is less than $6.0 \mathrm{~s}$.

Although the IBS series attenuated the amplitude and high frequency components of the EEG record, this change lasted only several minutes. When the animals were tested $24 \mathrm{~h}$ later, a time when all animals were still exhibiting clear evidence of inhibition, the baseline EEG record appeared normal with respect to amplitude and frequency. Therefore, although it is conceivable that the altered EEG is reflecting the process producing the long-term inhibition, it does not persist in a form easily detectable in the EEG records for more than several minutes. It is possible that a spectral analysis of the EEG record would have revealed some longlasting change not evident in our records.

It was generally reported that in fully kindled animals an "all-or-none" principle seems to operate in that stimulation evoking any afterdischarge normally triggers a stage 5 convulsion of normal intensity and duration $(14,15)$. It should be noted, however, that after an IBS series, the animals sometimes exhibited a stage 1 or 2 convulsion and a brief afterdischarge in response to stimulation suggesting that the mechanisms underlying the buildup of afterdischarge and its propagation in kindled animals had been altered. We also observed several instances during an IBS series when stimulation produced an afterdischarge which was not accompanied by behavioral signs of a motor convulsion. Although we cannot explain these results at present, it might be noted that dissociations of afterdischarges from motor convulsions in kindled animals was observed previously. For example, the GABA agonist $\gamma$-acetylenic GABA (GAG) was recently reported to produce a dissociation of electrographic seizures and motor convulsions in kindled animals (11).

It is of considerable interest that several animals exhibited a brief period of hyperresponsiveness and aggressiveness after an IBS series. In some animals the heightened emotional response is equivalent to that observed 